\title{
Research on Resources Integration of Urban Distribution System based on System Dynamics
}

\author{
Chen Liang ${ }^{1}$ and Dong Mu ${ }^{1,2}$ \\ ${ }^{1,2}$ Beijing Jiaotong University, Beijing 100044, China; \\ ${ }^{1}$ Beijing Wuzi University, Beijing 101149, China \\ ${ }^{1}$ liangchen727@sina.com, ${ }^{2}$ mueast@163.com
}

\begin{abstract}
Resource of urban distribution system is a typical distributed network structure and the goal to integrate the resources of urban distribution system is to maximize the system value. This paper carries out an in-depth analysis on urban distribution system by the methodology of System Dynamics. The modeling and computer simulation are given after the dissection of system boundary, main system factors and system causality. The simulation shows that, to achieve the desired integration level and to improve the resources owners' efficiency, optimal the exogenous environment and introduce more favorable polices are both feasible, but to the other two factors, physical resources factor only effect the resources owners' efficiency and the coordination mechanisms factor impact none.
\end{abstract}

Keywords: urban distribution system, resources integration, system dynamics, simulation

\section{Introductions}

With the rapid changing of modern production modes, commercial activities and consumption patterns, urban distribution is getting more and more important, and it has already became an indispensable part of urban economic system. In the past few years, urban distribution system is gaining independence from urban logistics system gradually, it showed significant system characteristics in nowadays. As a key component of urban distribution system, urban distribution resources refer to all the imputing things which aim at achieving urban distribution system goal, it not only includes the narrowly speaking resources, such as capital, technology, knowledge, information, human resources, premises and facilities, but also includes the generalized resources, such as distribution organization, distribution institution and the relevant laws or regulations.

In current urban distribution system, there are many prominent problems, such as vicious cycle between urban distribution resources inputs and its efficiency, unclear situation of resources owners' microscopic behavior and the immature of urban distribution market mechanism, these phenomenon have restricted and now is restricting the developing of the urban distribution system seriously. In addition, urban environmental pollution, urban traffic congestion, traffic accidents and other environment problems are inextricably linked with urban distribution activities, these problems have become a serious impediment to the overall development of modern urban.

By reasonable configuration of decentralized urban distribution resources, we can achieve the organic integration and " $1+1>2$ "effect goal, and in this way we can improve our urban living environment. So research in the resources integration issue of urban distribution system has an important practical significance.

\section{Literature Review}

According to the literature review, we know that urban distribution system evolved from urban logistics system. Professor Eiichi Taniguch in Kyoto University is one of the earliest 
who introduced the concept of urban logistics; he thought urban logistics is an optimal process of enterprise logistics and transportation activities within urban system, which considering the traffic environment, traffic congestion, energy waste and some other relevant factors [1, 2]. As an comprehensive system, urban logistics system includes lots of subsystems from its function perspective, such as transportation subsystem, warehousing subsystem, circulation processing subsystem, handling subsystem, distribution subsystem, packing subsystem, logistics information system, logistics equipment subsystem and logistics consulting subsystem [3]. To urban distribution subsystem, ZT WANG thought the decentralization characteristics of urban distribution system resources determine they must be self-organized in a certain way, form different distribution networks, and he also thought that the integration effect between these resources represents the level of urban distribution system [4]. However, current researches on this issue just focus on the definition of concept and system optimization angles, there aren't any literature research on the urban distribution system from the resources integration angle. The relevant research paper can be summarized as follows: AM DENG et al., made an in-depth research on the connotation, component, function and optimization goal of urban distribution system, they introduced the research framework, basic methods and specific steps of urban distribution system optimization based on urban distribution system's function and appraisal [5]. Z WANG et al. deeply dissect the urban distribution system, and they proposed the architecture and functional modules of urban distribution system by the methodology of terminal management [6]. YC YAN introduced the real-time urban distribution system solutions of $\mathrm{Xi}$ 'an city, and he also designed the functional modules of this solution in his paper [7]. JJ ZHU and Y PU discussed the construction problem of hub urban distribution system, they discussed it from the following three aspect: the construction of integrated transport system, the construction of efficient logistics information system and the construction of urban distribution nodes [8]. Crainic Teodor Gabriel et al., emphasized the relationship of urban distribution system and the urban economic development, they introduced a two-track distribution structure to evaluate and plan the urban distribution system [9]. But as we described above, there are little research on urban distribution system from resources integration aspect, there are lack of valuable academic research literature.

Traditional researches on resources integration are mainly by operation research method, they solve this problem by constructing accurate models, but these models have been already proved faulty in actions, such as high accuracy requirement of data, large amount of computation of models and so on. What's more, it is very difficult to trace the system variables' logic and number relationships of this method [10]. Studying the resources integration of urban distribution system by operation research method is not a very good way.

System Dynamics is a good way to avoid these defects of traditional operation research methods, many scholars have introduced system dynamics into logistics research, and they have achieved fruitful research results [11-13], these studies have demonstrated the applicable. Thus, we research the resources integration problem of urban distribution system by system dynamics method in this paper. This paper constructed the model and made a computer simulation after the dissection of system boundary, main system factors and system causality.

\section{System Dynamics Model of Urban Distribution System Resources Integration}

\subsection{Determine the System Boundary}

According to system dynamics theory, system boundary is directly related to our model, it is very important to determine an appropriate system boundary [14]. To construct the resources integration system dynamics model of urban distribution system, the first thing is to determine the system boundary, and the system boundary means which part should be 
included and which part should not be included in the system. We proposed the structure of urban distribution system in Figure 1, considering the major factors of urban distribution system.

As we can see from Figure 1, there about 10 factor which is listed as follows: physical distribution resources level (such as information technology), generalized distribution resources (such as relevant regulations), exogenous environment factors (such as residential environment requirement), specialized distribution service level, the efficiency of urban distribution system resources integration, the cost of urban distribution system resources integration, urban distribution system entity resources inventories, the integration level of urban distribution system resources, the resources owners' efficiency of urban distribution system and so on.

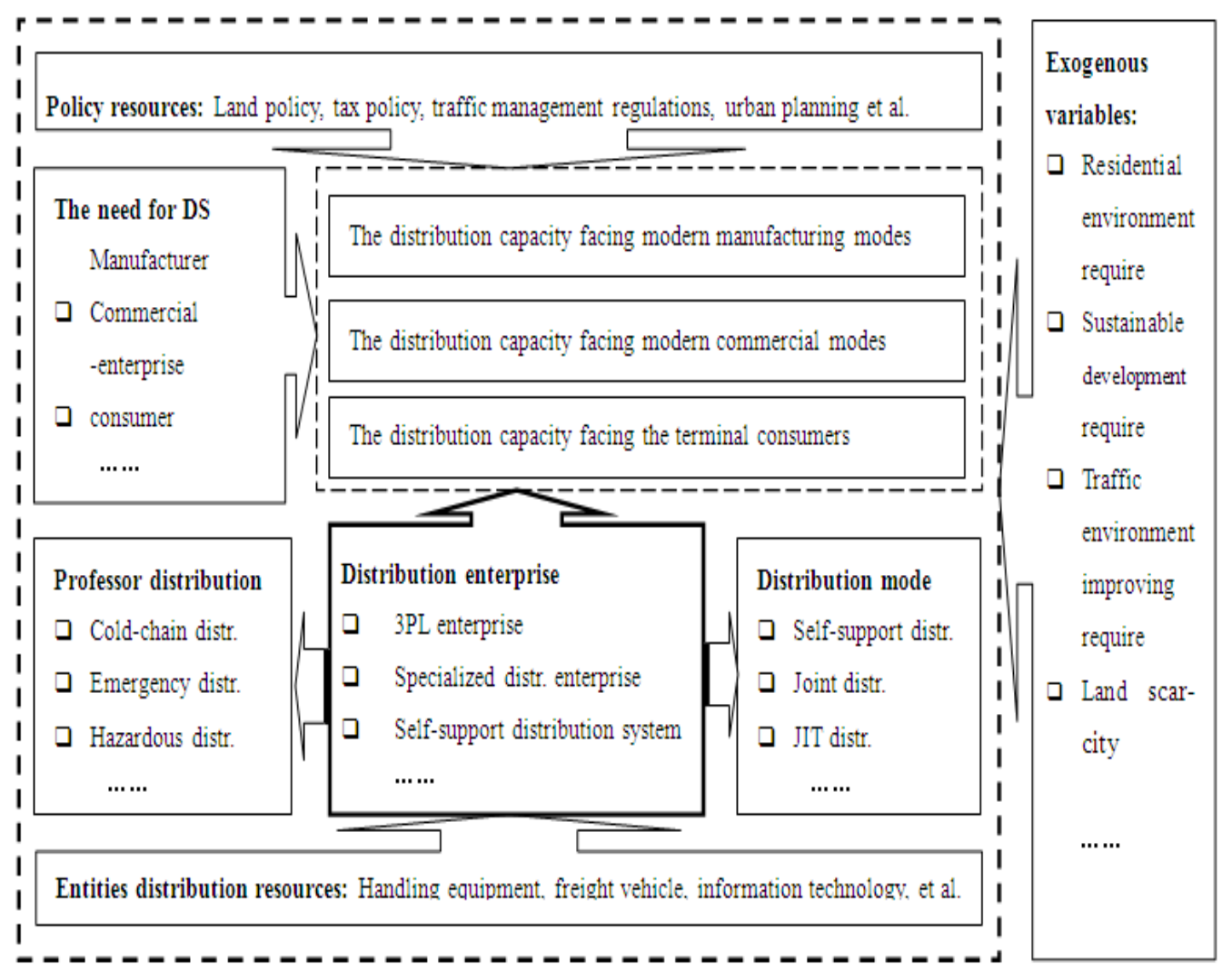

Figure 1. The Structure Diagram of Urban Distribution System (DS)

\subsection{System Dynamics Causality and Flow Diagram Analysis}

All factors in the system are connect with each other, influence with each other and make cause and effect with each other. In system dynamics theory, the relationship between the factors can be summarized as causation, and just as this causal interaction, it eventually forming the system function and behavior. So we carried out the causality analysis and the specific causal relationships can be seen in Figure 2.

In the causal diagram, there are 4 main feedback loops which are listed as below:

1) Urban distribution system entity resources inventories---Urban distribution system resources integration costs---The requirement of resources owners---The requirement of urban distribution system resources integration---The integration level of urban distribution system resources---Urban distribution system entity resources inventories;

2) The efficiency of urban distribution system resources---The efficiency of city distribution system resources owners---The requirement of urban distribution resources 
owners---The integration amount of urban distribution resources---Urban distribution system entity resources inventories ---The efficiency of urban distribution system resources;

3) The integration efficiency of urban distribution system resources---The differs between integration goal and the actual integration level--- The requirement of urban distribution system resources integration---The integration level of urban distribution system resources---The integration amount of urban distribution resources---The efficiency of urban distribution system resources integration;

4) The integration level of urban distribution system resources--- Urban distribution system entity resources inventories---Urban distribution system resources integration costs---The integration level of urban distribution system resources.

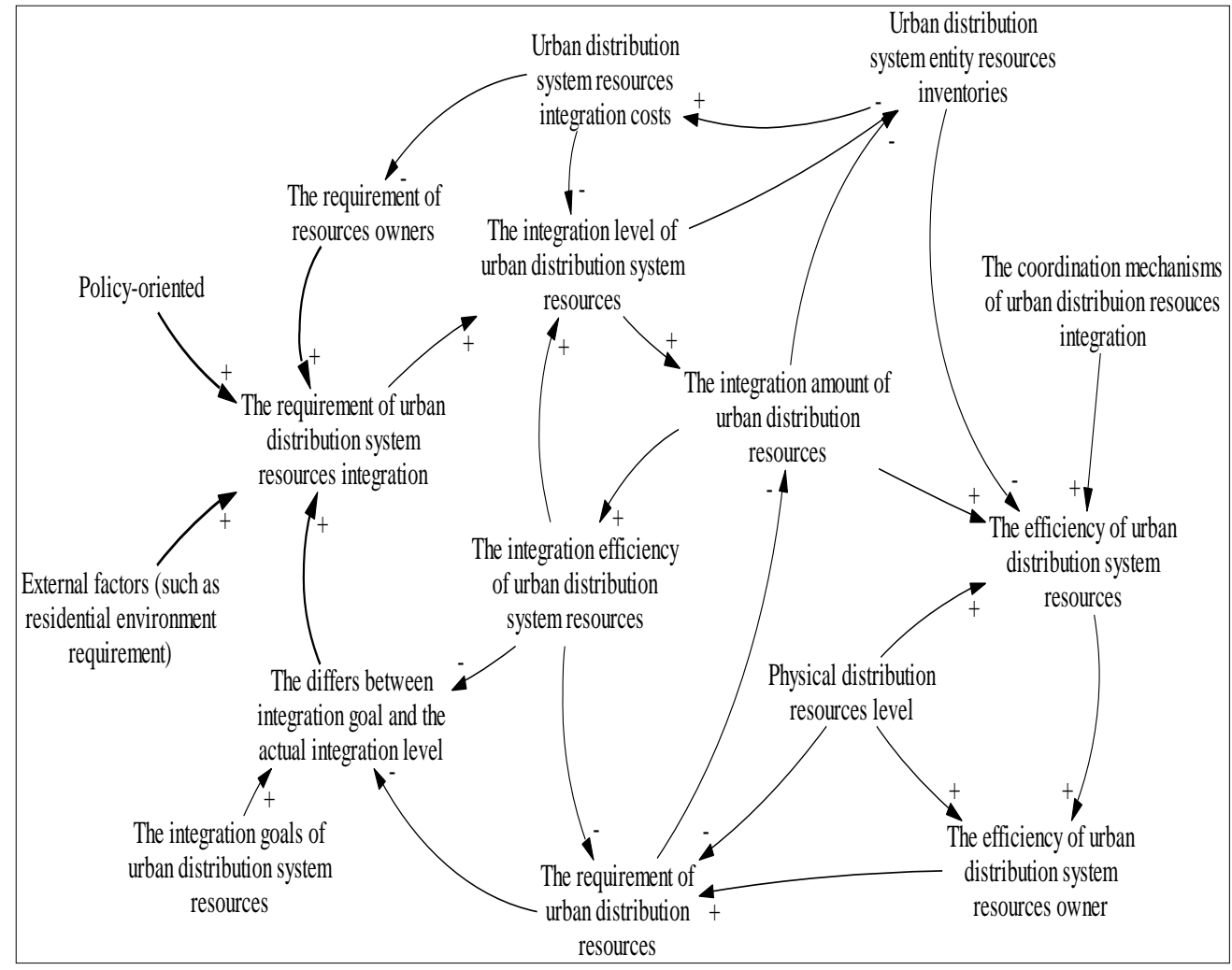

Figure 2. The System Dynamics Causality Diagram

\subsection{Establish the System Dynamics Equation}

This system dynamics model contains 4 stock variables, which are urban distribution system entity resources inventories, the efficiency of urban distribution system resources owner, the efficiency of urban distribution system resources integration and the integration level of urban distribution system resources respectively. To satisfied these stock variables, we set 5 flow variables in this model, which are the growth speed of urban distribution system resources integration revenue, the growth speed of urban distribution system resources integration costs, the integration speed of urban distribution resources, the efficiency variation of urban distribution resources and the variation of urban distribution resources respectively. According to these settings, we construct the system dynamics flow diagram of urban distribution system resources integration in VENSIM, which can be seen in Figure 3.

The relationship of the main variables involved in this model is listed as follows:

1) The urban distribution system entity resources inventories = (The demand of urban distribution resources - The expectation variation of urban distribution resources 
integration) * (The coordination mechanisms of urban distribution system resources integration * The integration level of urban distribution system resources * Exogenous environment factor) / (The coordination mechanisms of urban distribution system resources integration + The integration level of urban distribution system resources + Exogenous environment factor);

2) The efficiency of urban distribution system resources owner $=($ Market price + The level of physical resources + The efficiency of urban distribution system resources + The integration level of urban distribution system resources) * The urban distribution system entity resources inventories;

3) The integration level of urban distribution system resources = Exogenous environment factor * "Policy-oriented" * (2005(any constant which is greater than 2000)-The expectation variation of urban distribution resources integration);

4) The efficiency of urban distribution system resources $=$ The level of physical resources $*$ The coordination mechanisms of urban distribution system resources integration $*$ The integration level of urban distribution system resources $*$ Exogenous environment factor;

5) The expectation variation of urban distribution resources integration $=$ The demand of urban distribution resources -The expectation of urban distribution resources integration;

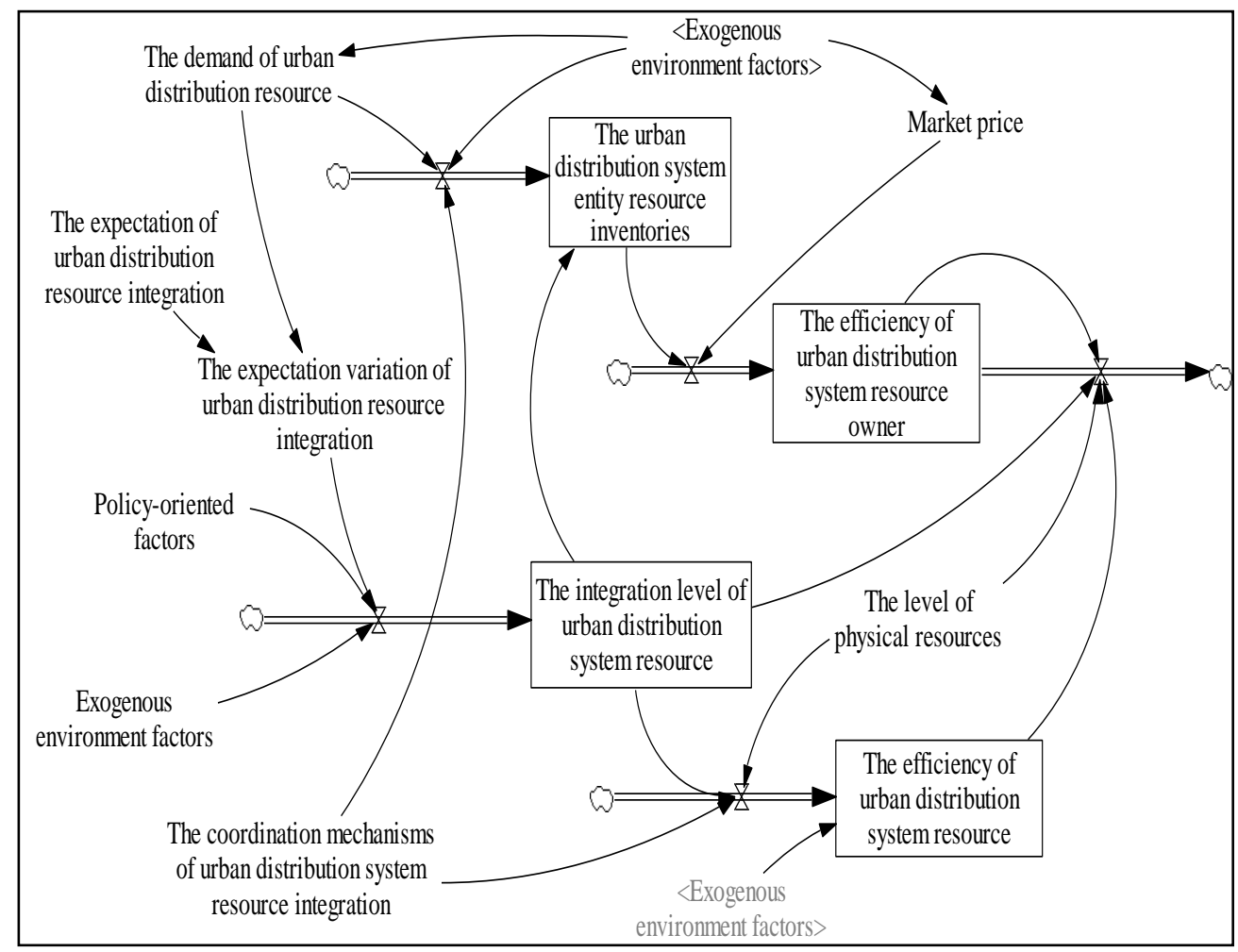

Figure 3. The System Dynamics Flow Diagram

\section{Examples Simulation and Results Analysis}

The integration level of urban distribution system resources and the efficiency of urban distribution system resources owners are related to many factors, which can be seen from the above causality diagram. We can obtain the corresponding results by adjusting the key factors in the model.

1) Exogenous environment factor 
The number of exogenous environment factor falls from 1 to 9 , and the larger the number was, the better exogenous environment (The higher the exogenous environment factor was, the higher demand comes of the public, and it could promote the integration of distribution system resources). As we can see from Figure 4, the efficiency of urban distribution system resources owners and the integration level of urban distribution system resources increases with the increase of exogenous environment factor.

2) Policy-oriented factor

The number of policy-oriented factor falls from 1 to 9 , the large the number was, it represents that the government will introduce more favorable policy. As we can see from Figure 5, on the condition of other factor being constant, the higher the policy-oriented factor is, the higher efficiency of urban distribution system resources owner and integration level of distribution system resources. In condition, compared with other factors, we can obtain that the policy-oriented factor affect most to the resources integration of urban distribution system.
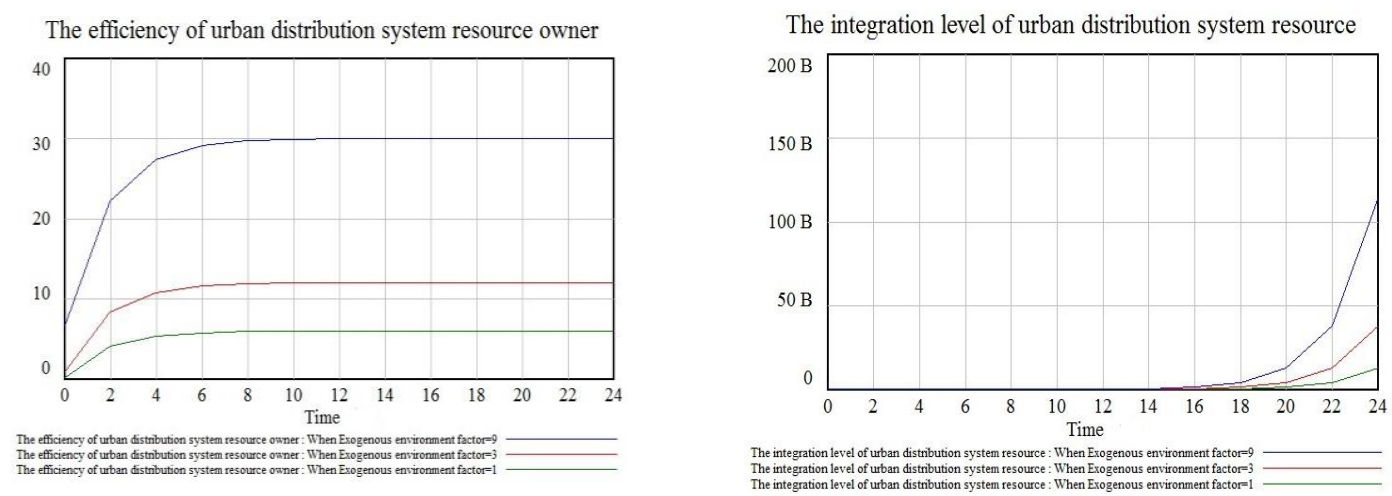

Figure 4. The Impact of Exogenous Environment Factor
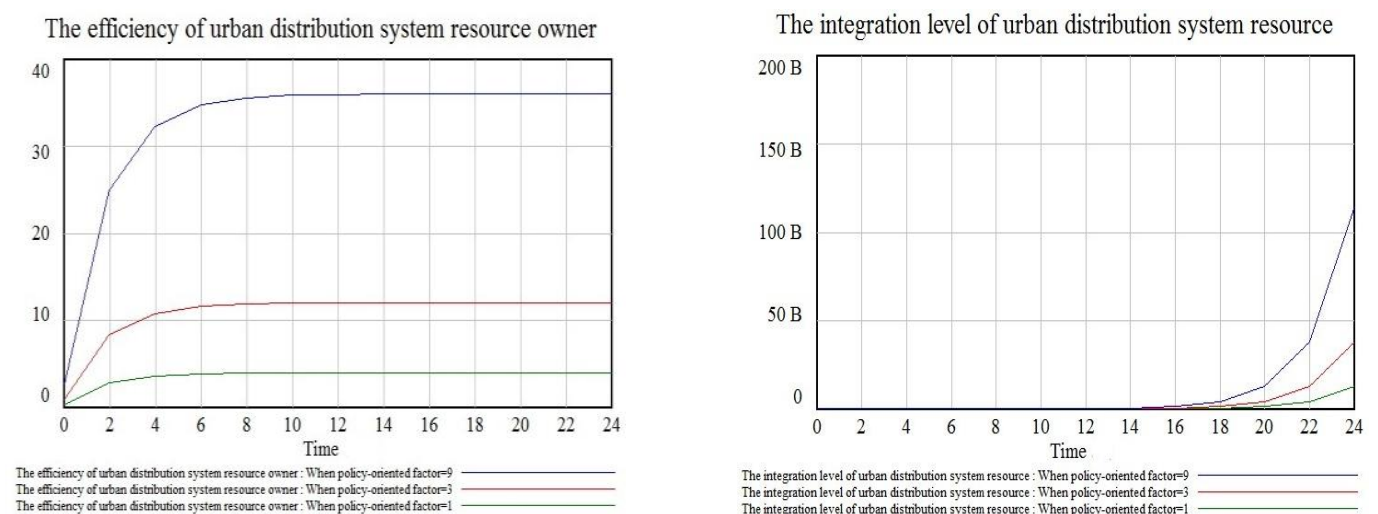

Figure 5. The Impact of Policy-oriented Factor

3) The coordination mechanisms factor

The number of coordination mechanisms factor also falls from 1 to 9 . As we can see from Figure 6, the efficiency of urban distribution system resources owners and the integration level of urban distribution system resources both have no relationship with the coordination mechanisms. So, increase or decrease the coordination mechanisms have no effect on the resources integration of urban distribution system.

To this situation, we can explain it as follows: Urban distribution system resources integration is a macroscopic concept, and the coordination mechanisms represent the 
coordination level between the resources owners, which belongs to a microscopic scale. The coordination mechanisms represents the distribution of efficiency between the resources owners, it has no effect on the overall efficiency.

4) The physical resources factor

The number of coordination mechanisms factor also falls from 1 to 9 . As we can see from Figure 7 the efficiency of urban distribution system resources owner increases with the number of physical resources level, but the integration level of urban distribution system resources have no relationship with this factor. So, if we want to increase the efficiency of urban distribution system resources owners, we can increase the physical resources level, but if we want to increase the resources integration level of urban distribution system, increase or decrease the physical resources level wouldn't change anything.
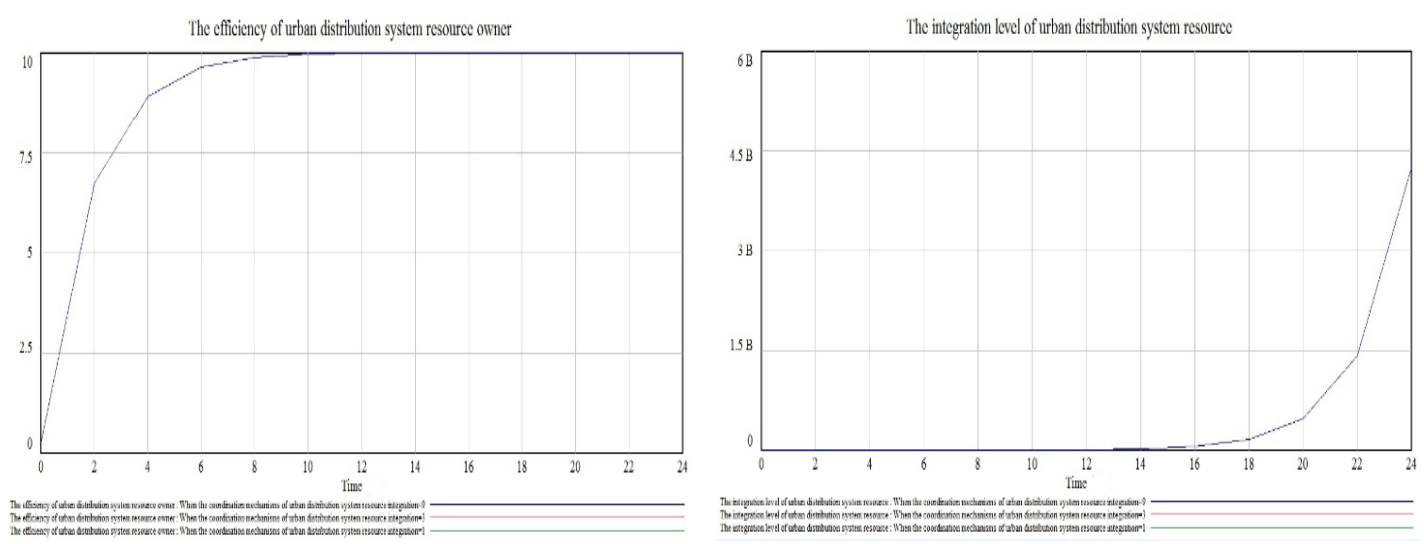

Figure 6. The Impact of Coordination Mechanisms Factor

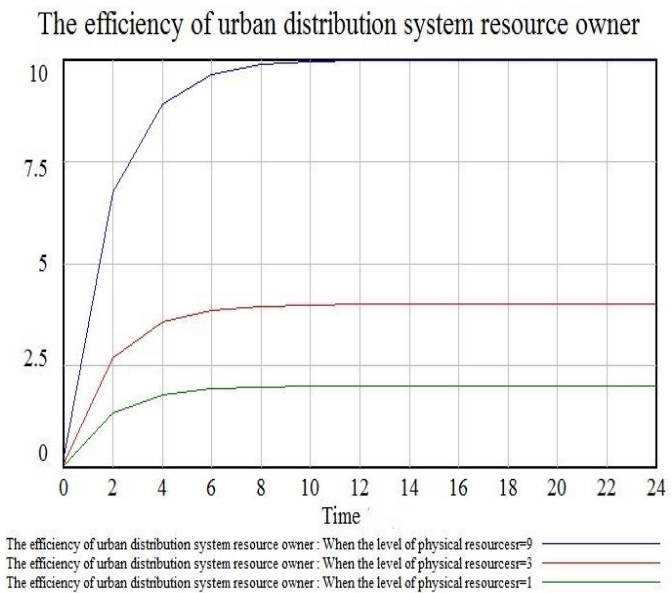

The integration level of urban distribution system resource

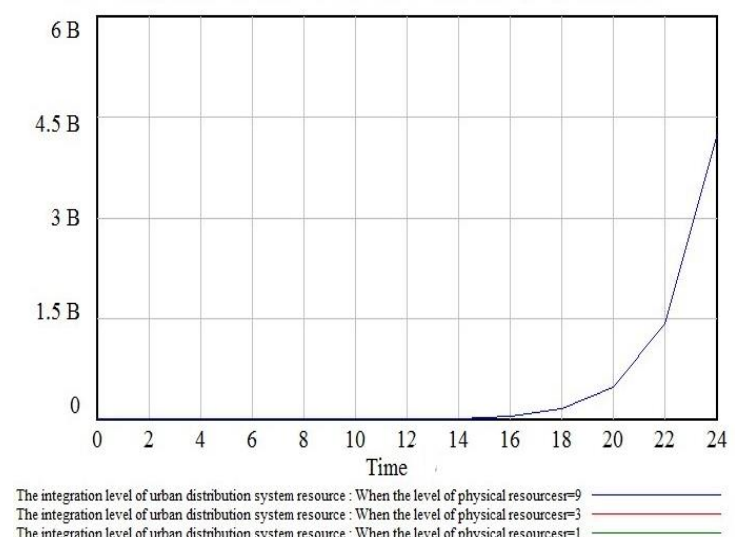

Figure 7. The Impact of Physical Resources Level

\section{The Conclusions and Proposals}

This paper discusses the resources integration problem of urban distribution system. The aim of this paper is to explorer the integration mechanism of urban distribution system resources. To solve this problem, this paper studies on this issue by system dynamics mythology. This paper dissected the system boundary first, and then discussed the main system factors and system causality, it construct the model and made a computer simulation at the end of this paper. 
As we can see from the system dynamics simulation, integrate the resources of urban distribution system can help increase the owners' efficiency of urban distribution system resources, so as to improve the value of the urban distribution system. In the implementation process of urban distribution system resources integration, we first need to strive for the government policy support, promoting effective integration of urban distribution system resources. Then, we need to create a favorable exogenous environment condition, strive for the public awareness and support of urban distribution system resources integration. At last, we can also improve the recourses owners' efficiency of urban distribution system by improving the physical resources level. In addition, the simulation shows the coordination mechanisms factor have no effect on the resources integration of urban distribution system.

\section{Acknowledgements}

The research work was supported by National Natural Science Foundation of China under Grant No. 71132008, Funding Project for Academic Human Resources Development in Institutions of Higher Learning under the Jurisdiction of Beijing Municipality and the Scientific Research Bases- Science and technology innovation platform of Beijing Wuzi University.

\section{References}

[1] E. Taniguchi, M. Noritake and T. Yamada, "Optimal size and location planning of public logistics terminals", Transportation Research Part E, vol. 35, no. 3, (1999), pp. 207-222.

[2] E. Taniguchi, "City Logistics: Network modeling and intelligent transport system", Pergamum Publishing, (2001).

[3] L. Y. Zhou and D. Mu, "Research on Synergetic Evolution of Regional Logistics System", Journal of Beijing Jiaotong University (Social Sciences Edition), vol. 8, no. 2, (2009), pp. 26-28.

[4] Z. T. Wang, "Logistics Engineering Research", Beijing: Capital University of Economics Press, (2004).

[5] A. M. Deng, S. M. Wang and L. J. Wang, "City Logistics Distribution System Optimization Research", Journal of Wuhan University of Technology (Transportation Science \& Engineering), vol. 30, no. 3, (2006), pp. 481-484.

[6] Z. Wang and J. Shi, "A model for urban distribution system under disruptions of vehicle travel time delay", Intelligent Computation Technology and Automation, 2009. ICICTA'09. Second International Conference on. IEEE, vol. 3, (2009), pp. 433-436.

[7] Y. C. Yan, "Design of Functional Module of Urban Logistics Real-time Distribution System", Logistics Technology, vol. 31, no. 11, (2012), pp. 412-414.

[8] J. J. Zhu and Y. Pu, "Construction of Urban Logistics Distribution System in Hub City", Logistics Technology, vol. 07, (2013), pp. 16-19.

[9] T. G. Crainic, N. Ricciardi and G. Storchi, "Models for evaluating and planning city logistics systems", Transportation science, vol. 43, no. 4, (2009), pp. 432-454.

[10] H. A. Eiselt and C. L. Sandblom, “Operations research: a model-based approach”, Springer, (2012).

[11] Q. L. Gu and T. G. Gao, "Joint decisions for R/M integrated supply chain using system dynamics methodology", International Journal of Production Research, vol. 50, no. 16, (2012), pp. 4444-4461.

[12] A. A. Tako and S. Robinson, "The application of discrete event simulation and system dynamics in the logistics and supply chain context", Decision Support Systems, vol. 52, no. 4, (2012), pp. 802-815.

[13] L. Liu and X. Guan, "Study on the Risks of Mining Logistics System Based on System Dynamics Model", The 19th International Conference on Industrial Engineering and Engineering Management. Springer Berlin Heidelberg, (2013), pp. 919-928.

[14] X. Li, "Social System Dynamics", Shainghai: Fudan University Press, (2009).

\section{Authors}

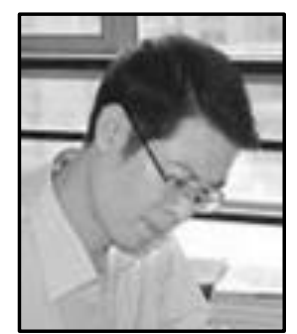

Chen Liang, He is a full instructor of Beijing Wuzi University (2005). He received his master degree in Management science and engineering from Beijing Wuzi University, and now he is working on his PhD degree in Beijing Jiaotong University science 2009. His current research interests include different aspects of logistics engineering and Artificial Intelligence and Systems dynamics. 


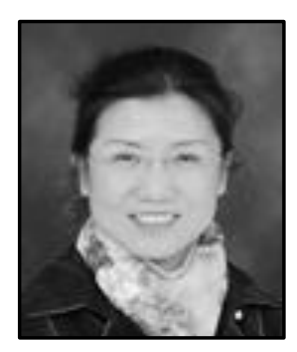

Dong Mu, She received her $\mathrm{PhD}$ degree in Business and Management School of Tongji University(1999). Now she is a full time professor at Management School of Beijing Jiaotong University since 2005. Her current research interests include different aspects of logistics and supply system engineering, regional logistics and environmental evaluation and system dynamics. 
International Journal of $u-$ and $\mathrm{e}-$ Service, Science and Technology Vol.6, No.6 (2013) 